\title{
A Method of Measuring TEC at Low Elevation Angles
}

\author{
FENG-RONG YEH ${ }^{1}$, KEN HUANG LIN ${ }^{1}$ and KUNG CHIE YEH ${ }^{1}$ \\ (Manuscript received 4 December 1994, in final form 15 March 1995)
}

\begin{abstract}
A radio wave may suffer severe refractional effects at low elevations. Such effects will make the determination of the total electron content (TEC) inaccurate. Recently developed tomography techniques applied to ionospheric imaging would require accurate TEC measurements from overhead to the lowest possible elevation for a good reconstruction. It is, therefore, desirable to develop a method that will give accurate TEC measurements even at low elevation angles. One method that uses the difference of the TEC measured at two lower frequencies as a correction of the TEC measured at a higher frequency is proposed. The model study here shows the corrected TEC has a three-digit accuracy for all elevations. At low elevations the troposphere may also influence the radio path. The attempt here to extract the tropospheric information from the phase data shows only a moderate success for the atmospheric models studied.
\end{abstract}

(Key words: TEC, Radio beacon experiment, Dispersive Doppler, Phase difference measurement)

\section{INTRODUCTION}

The radio beacon experiment is one of the oldest satellite-based space experiments (Davies, 1980). In this experiment, an orbiting satellite emits a number of frequencies which are usually harmonically related. These emissions are then received on the ground. The characteristics of the received emissions are modified by the intervening propagation medium, i.e. the ionosphere and the troposphere. By measuring the modifications on the received radio waves, such as the amplitude, phase, polarization, direction of arrival, Doppler, etc., some infonnation about the propagation medium can be obtained. When the medium has a spatial scale smaller than the Fresnel scale, the radio wave suffers a single or multiple scattering, resulting in the scintillation phenomenon (Yeh and Liu, 1982; Aarons, 1982; Bhattacharyya et al., 1992). These studies have been used to investigate the characteristics of ionospheric irregularities, the effects on transionospheric radio signals and the geomorphology of irregularities on a global basis. On the other hand, when the medium has a spatial scale

1 Department of Electrical Engineering, National Sun Yat-Sen University, Kaohsiung, Taiwan, R.O.C. 
much larger than the Fresnel scale, the radio wave suffers only the refractional effects with the associated phase changes. In this case, the proper processing of the received signal yields the integrated election density, also called the total electron content (e.g. Garriott et al., 1970; Davies, 1980). Extensive discussions on these subjects can be found in the proceedings of a series of Radio Beacon Symposia (e.g. Lee, 1992). In almost all past studies, the satellite elevation angles are confined to above about 20 since it is known that the ray bending at low elevations can be substantial. For example, at $40 \mathrm{MHz}$ an early analysis of some satellite data has shown that an error in the TEC can be as large as $15 \%$ (Yeh and Swenson, 1961) even when the orbiting satellite is near its point of closest approach. The effect caused by ray bending must therefore be properly taken into consideration. The low elevation angle measurements are of interest for at least two reasons. Firstly, only at low elevation angles can the tropospheric ray bending become significant; therefore, this is the regime to get tropospheric information if it is at all possible (Gorbunov and Gurvich, 1993). In fact, experimental measurements have shown that gigahertz scintillation at low elevations can be caused by turbulence in the troposphere (Fang and Liu, 1983). Secondly, in ionospheric tomography applications, it is desirable to obtain the TEC from overhead to as low an elevation angle as possible, for a good reconstruction of the two-dimensional image (Yeh and Raymund, 1991). Motivated by these two interests, the authors undertake this investigation.

In section 2 the ray tracing formulas used in the calculations are briefly reviewed. These formulas are very much simplified for a spherically stratified atmosphere. Some results are presented in several figures. Using these results, it is shown in section 3 how the phase difference can be processed for accurate TECs even at low elevation angles and the marginal information about the troposphere that is obtainable. Section 4 concludes the paper.

\section{RAYS IN A SPHERICALLY STRATIFIED ATMOSPHERE}

This study involves the tracing of radio rays from a given transmitter to a fixed receiver or vice versa. For simplicity, the ionospheric medium is assumed to be isotropic. Since the propagation medium is inhomogeneous, the ray tracing must involve a "homing" feature. For a radio frequency in the gigahertz range, the wavelength is only $2 \mathrm{~cm}$. If the ray tracing is to introduce a phase error of not more than a few degrees, the homing must be accurate to at least $10^{-3} \mathrm{~m}$. This kind of accuracy requires repeated ray tracing and can thus increase numerical computations fairly substantially. In order to keep computations to a minimum, and yet not to lose the physics of the problem, a spherically stratified atmosphere is assumed. Each ray propagating in such an atmosphere, as shown in Figure 1, follows Bouger's rule:

$$
a \sin \alpha_{0}=r n(r) \sin \alpha=a \cos \beta_{0},
$$

where $a$ is the Earth's radius, $r$ is the geocentric distance to a point on the ray, $n$ is the refractive index, $\alpha$ is the incidence angle which becomes $\alpha_{0}$ at the lower boundary of the ionosphere, and $\beta_{0}$ is the elevation angle. When (1) is applied to the differential ray path $d s$ of Figure 1:

$$
d s=\frac{n r}{\sqrt{n^{2} r^{2}-a^{2} \cos ^{2} \beta_{0}}} d r .
$$

Using (2) or its equivalent, expressions for the ground range $D$, the optical path $p$ and the group path $p^{\prime}$ can all be found as, respectively (Lawrence et al., 1964): 


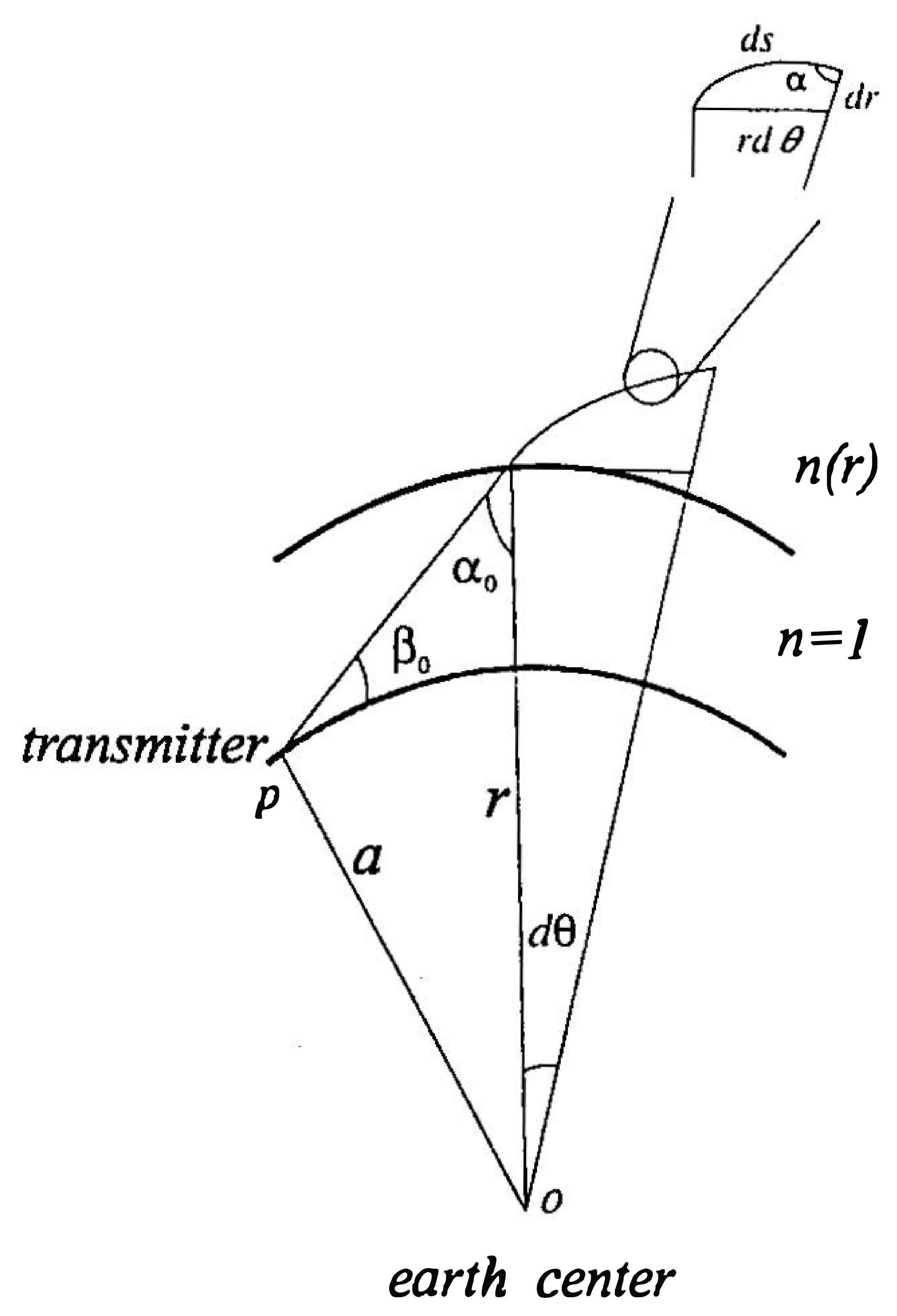

Fig. 1. Depicting the ray geometry in a spherically stratified atmosphere.

$$
\begin{aligned}
& D=\int_{a}^{r} a d \theta=\int_{a}^{r} \frac{a^{2} \cos \beta_{0}}{r \sqrt{n^{2} r^{2}-a^{2} \cos ^{2} \beta_{0}}} d r \\
& p=\int_{a}^{r} n d s=\int_{a}^{r} \frac{n^{2} r}{r \sqrt{n^{2} r^{2}-a^{2} \cos ^{2} \beta_{0}}} d r
\end{aligned}
$$

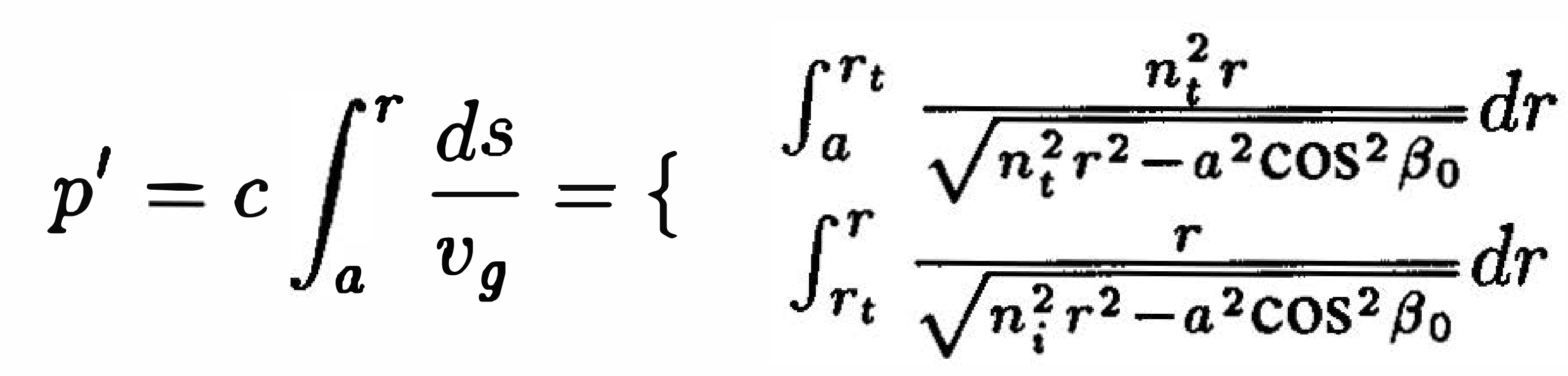

It should be noted that the phase is related to the optical path simply by $\phi=(\omega / c) p$. The group velocity $v_{g}$ is given by $v_{g}=c / n_{t}$ in the troposphere, and by $v_{g}=c n_{i}$ in the ionosphere. Thus, (5a) applies to the troposphere where $r_{t}$ is the top of the troposphere, and (5b) applies to the ionosphere.

Before rays can be traced, a model atmosphere must be postulated. The real troposphere can be quite complicated (Hall, 1979). In this investigation a simple exponential pressure is assumed.

$$
P(r)=P(a) \exp [-(r-a) / H]
$$

where for numerical computations the surface pressure $P(a)$ is assumed to be $1013.25 \mathrm{mb}$, and the scale height $H$ is taken to be $8.4 \mathrm{~km}$. Several scale heights above the ground, the 
exponential atmosphere decays rapidly to negligible values. In real atmospheres, there are overlaying middle and upper atmospheres. But these higher atmospheres have very little effect on radio waves; they can be justifiably ignored, as is done in this paper. For the ionosphere, the electron density $N$ has the Chapman model:

$$
N(r)=N_{m} \exp \left(1-x-e^{-x}\right) / 2,
$$

where the peak electron density $N_{m}$ takes a value $1.25 \times 10^{12}$ electrons $/ \mathrm{m}^{3}$, and the reduced height $x=\left(r-r_{m}\right) / H_{c}$ has $r_{m}=6696 \mathrm{~km}$ and $H_{c}=50 \mathrm{~km}$. Since the modeled troposphere (6) and ionosphere (7) are pratically nonoverlapping, the refractive index for the whole atmosphere can be written as:

$$
n(r, f)=1-\frac{80.6 N}{2 f^{2}}+\frac{A P \times 10^{-6}}{T}+H O(r, f)
$$

Numerically, $\mathrm{A}=77.6 \mathrm{~K} / \mathrm{mb}$, and $\mathrm{T}=288^{\circ} \mathrm{K}$. There are four terms on the right hand side of (8) that contribute to the refractive index. The first term represents a contribution from free space, the second term represents a first order contribution from the ionosphere, the third term represents that from the dry troposphere, and the fourth term represents all higher order contributions from the ionosphere. When (8) is inserted into (3) or (4), many terms result. The ground range $D$ is used to find the initial elevation angle $\beta_{0}$ of a ray that will "home" to the satellite. The satellite, in a circular orbit, is assumed to be at the height of $1100 \mathrm{~km}$. The initial line-of-sight elevation angle for a given satellite position is continually modified until $D$ is within $10^{-7} \mathrm{~km}$. Achieving this accuracy or better implies a successful homing of the ray. The $\beta_{0}$ of the homed ray is then used in (4) to find the optical path. From (4), it is found that:

$$
p=p_{r p}+p_{i}+p_{t}+p_{H O},
$$

where the free space term along the ray path is:

$$
p_{r p}=\int_{r a y p a t h} d s
$$

the first order ionospheric term is:

$$
p_{i}=-\int \frac{80.6 N(r)}{2 f^{2}} d s
$$

the tropospheric term is:

$$
p_{t}=\int \frac{A P(r)}{T} \times 10^{-6} d s
$$

and the higher order term is:

$$
p_{H O}=\int H O(r, f) d s
$$


In the four terms of (9), the free space term is the largest. To assess the effects caused by ray bending, the free space optical path difference is defined as:

$$
p_{f}=p_{r p}-p_{s p}
$$

where

$$
p_{s p}=\int_{\text {straight line path }} d s
$$

The total medium effects outside the straight line path are defined as the deviation optical path $p_{d}$,

$$
\begin{aligned}
p_{d} & =p-p_{s p} \\
& =p_{f}+p_{i}+p_{t}+p_{H O} .
\end{aligned}
$$

The magnitudes of $p_{i}, p_{d}, p_{t}, p_{f}$ and $p_{H O}$ are separately computed as a function of straight-line elevation angles from $0^{\circ}$ to $19^{\circ}$ for frequencies from $200 \mathrm{MHz}$ to $1.6 \mathrm{GHz}$ at $200 \mathrm{MHz}$ increments(Yeh, 1994). But in the interest of saving journal space, the results only for $200 \mathrm{MHz}$ [see Figure 2(a)] and for $1.6 \mathrm{GHz}$ [see Figure 2(b)] are shown. At $200 \mathrm{MHz}$, the ionospheric optical path $p_{i}$ is by far the largest term. This shows why the high VHF and low UHF frequencies are ideal in the measurement of the TEC. The tropospheric contribution $p_{t}$ is minor even for grazing rays. As the frequency increases, the tropospheric contribution becomes increasingly important, especially at low elevation angles. At $800 \mathrm{MHz}, p_{t}$ actually exceeds $p_{i}$ at grazing. At $1.6 \mathrm{GHz}, p_{t}$ exceeds $p_{i}$ for elevation angles up to about $11^{\circ}$. In all the computations in this study, the excess path in free space caused by ray bending $p_{f}$ and the higher order ionospheric path $p_{H O}$ are negligible for all frequencies.

With the preliminary results computed in this section, an experimental technique to obtain the accurate TEC for low elevation angles and a method to obtain tropospheric information can be discussed.

\section{PHASE DIFFERENCE MEASUREMENTS}

The phase $\phi$ of a radio wave at an elevation angle $\beta_{0}$ and a frequency $f$ is related to the optical path $p$ by:

$$
\phi\left(\beta_{0}, f\right)=(2 \pi f / c) p\left(\beta_{0}, f\right)
$$

In actual measurements, it is the dispersive phase,

$$
\Delta \phi\left(\beta_{0}, f_{1}, f_{2}\right)=\phi\left(\beta_{0}, f_{2}\right) / m_{2}-\phi\left(\beta_{0}, f_{1}\right) / m_{1},
$$

that is measured. The dispersive phase is the phase difference of radio waves at two frequencies $f_{1}$ and $f_{2}$ with $f_{2}>f_{1}$ when both frequencies are brought down to a common reference frequency $f_{0}$ for comparison. Thus, $f_{1}=m_{1} f_{0}$ and $f_{2}=m_{2} f_{0}$. For a meaningful phase comparison, this requires that the transmitted beacon frequencies be harmonically related. Inserting (9) into (17) and substituting the result into (18), the dispersive phase becomes:

$$
\Delta \phi\left(\beta_{0}, f_{1}, f_{2}\right)=\frac{2 \pi f_{0}}{c}\left[\Delta p_{f}+\Delta p_{i}+\Delta p_{i}+\Delta p_{H O}\right]
$$


(a) For a radio frequency of $200 \mathrm{MHz}$

frequency: $200 \mathrm{MHz}$

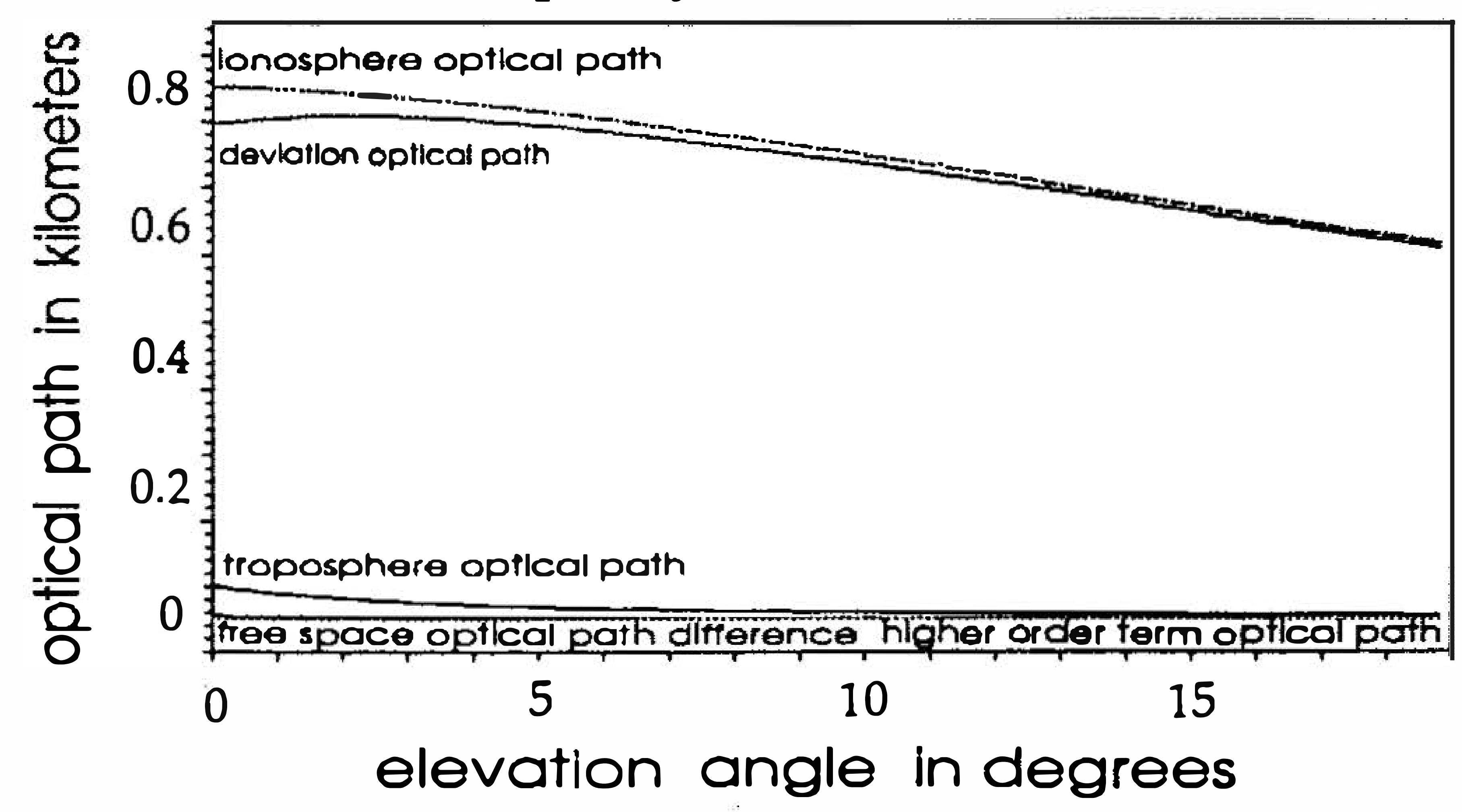

(b) For a radio frequeticy of $1.6 \mathrm{GHz}$ frequency: $1.6 \mathrm{GHz}$

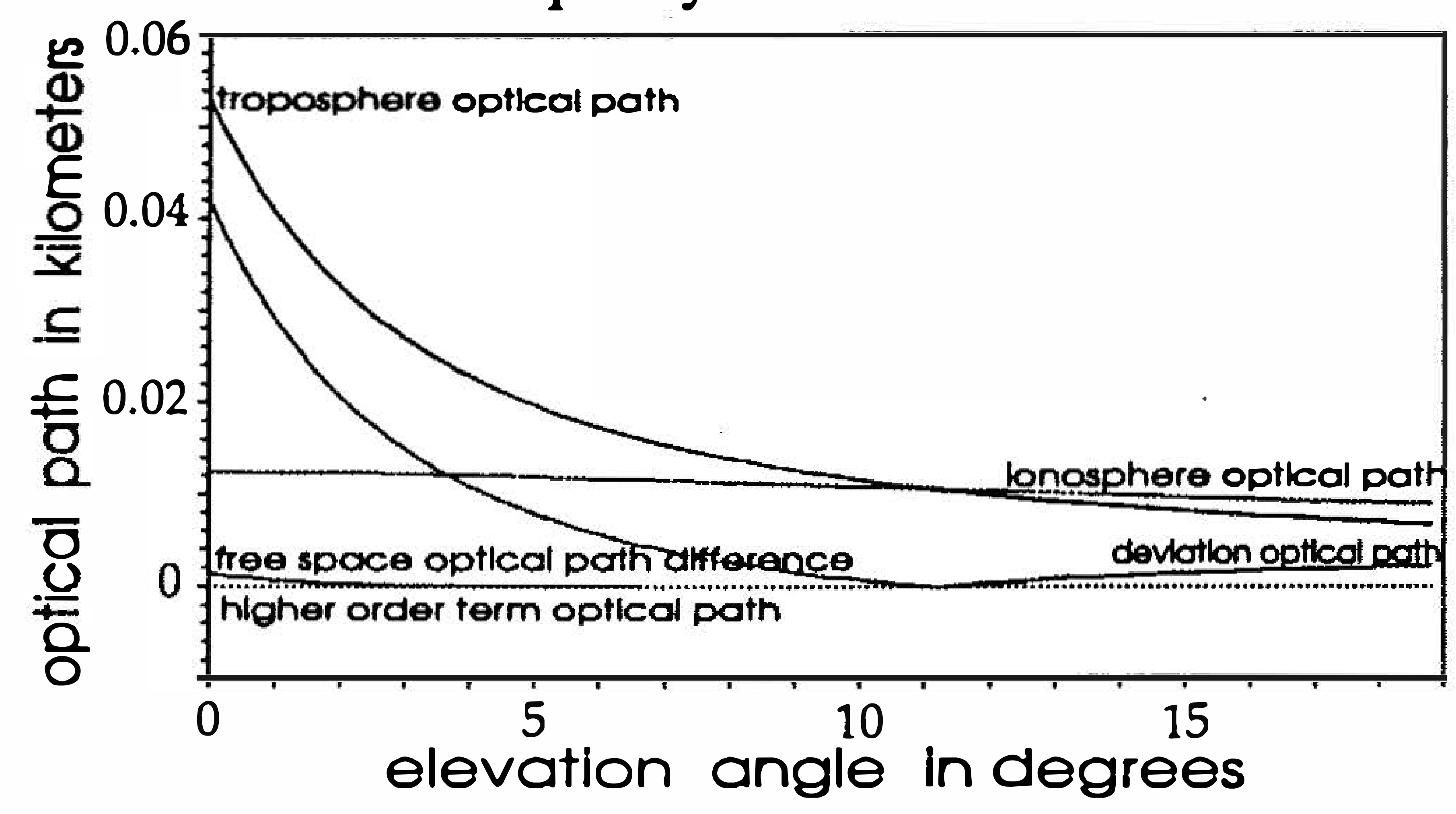

Fig. 2. The magnitudes of the optical paths $p_{i}, p_{d}, p_{t}, p_{f}$ and $p_{H O}$ as a function of line-of-sight elevation angle. The model troposphere and ionosphere are given in section 2. The satellite is at an altitude of $1,100 \mathrm{~km}$.

The free space straight line path has been subtracted out. The remaining four terms on the right hand side of (19) come from ray bending, the first order ionospheric effect, the tropospheric effect and the higher order ionospheric effect, respectively. Computational results of the previous section have shown that for high elevation angles $\left(\beta_{0}\right.$ larger than about $20^{\circ}$, the only important term in (19) is the ionospheric term. Making this assumption in (19) and substituting the ionospheric optical path (11) into the simplified (19) results in

$$
\operatorname{TEC}\left(\beta_{0}, f_{1}, f_{2}\right)=\frac{m_{1}^{2} m_{2}^{2}}{m_{2}^{2}-m_{1}^{2}} \frac{f_{0} c}{80.6 \pi} \Delta \phi\left(\beta_{0}, f_{1}, f_{2}\right)
$$

This is the usual working formula used to compute the oblique electron content from the measured dispersive phase (Davies,1980). This formula loses its accuracy when the elevation angle is less than about $20^{\circ}$.

\subsection{Corrected TEC}

The usual formula adopted by experimenters in calculating TEC from the dispersive phase data is (20). For elevation angles above $20^{\circ}$, it yields fairly accurate and reliable TEC 
values. However, for low elevation angles the formula (20) may produce inaccuracies. The major error comes from neglecting the tropospheric term in (19). In an attempt to correct for the tropospheric effect, an empirical formula is proposed:

$$
\left[\operatorname{TEC}\left(\beta_{0}\right)\right]_{c}=\operatorname{TEC}\left(\beta_{0}, f_{2}, f\right)-\operatorname{TEC}\left(\beta_{0}, f_{1}, f\right)+\operatorname{TEC}\left(\beta_{0}, f_{3}, f\right),
$$

where $f_{1}<f_{2}<f_{3}<f$. In this measurement, the difference of the TEC obtained from (20) at two lower frequencies is used to correct for the TEC obtained at a higher frequency. It should be noted that the tropospheric contribution of the optical path is approximately independent of frequency [see (21)]. On the other hand, the approximate formula (20) used in computing the TEC assumes an inverse frequency squared dependence. Thus, the error in the empirical formula (21) due to tropospheric contribution is largest at $f_{1}$ and smallest at $f_{3}$. As far as the tropospheric part of (21) is concerned, the situation can be described as partial cancellation of the largest tern by the sum of two smaller terms. Computations made for three cases are shown in Figure 3. Here, the relative error is the difference of the TEC computed by using (21) from the true TEC, divided by the true TEC. The true TEC for a given $\beta_{0}$ is obtained by integrating the Chapman modal ionosphere (7) along a straight line from the ground to the satellite. The frequency selections for the three cases are:

$\begin{array}{llll}\text { Case } 1 & f_{1}=200 \mathrm{MHz} & f_{2}=300 \mathrm{MHz} & f_{3}=400 \mathrm{MHz} \\ \text { Case 2 } & f_{1}=300 \mathrm{MHz} & f_{2}=400 \mathrm{MHz} & f_{3}=500 \mathrm{MHz} \\ \text { Case 3 } & f_{1}=400 \mathrm{MHz} & f_{2}=500 \mathrm{MHz} & f_{3}=600 \mathrm{MHz}\end{array}$

where $f=1.6 \mathrm{GHz}$ for all three cases. As seen from Figure 3, equation (21) has at least a two-digit accuracy for all three cases for the adopted atmospheric model. In particular, Case 1 has a three-digit accuracy for all elevation angles. Such an accuracy should satisfy the needs of tomographic applications.

\subsection{Tropospheric Scale Height}

Figures 2 have shown that for low elevation angles, the tropospheric effects can become important especially at gigahertz frequencies. The largest term in (19) is $\Delta p_{i}$ which can be estimated more accurately by using (21). With $\Delta p_{i}$ subtracted out, the remaining terms give:

$$
\Delta \phi_{t}=\frac{2 \pi f_{0}}{c}\left[\int n_{t} d s_{2}-\int n_{t} d s_{1}\right]
$$

where the higher order terms have been found to be small and, hence, have been dropped. The integration of the tropospheric refractive index $n_{t}$ for frequency $f_{1}$ has a differential element $d s_{1}$ and that for frequency $f_{2}$ has a differential element $d s_{2}$. The remaining dispersive phase difference (22) therefore depends on the difference of the tropospheric optical paths at two frequencies. The RHS of (22) can be numerically obtained. For the exponential model troposphere (6) adopted in this investigation, the LHS of (22) can be integrated and calculated as a function of the elevation angle. If the scale height is taken to be a parameter, its required value for the LHS equal to the RHS can thus be calculated. These calculated scale heights are marked by crosses in Figure 4 . This shows that the technique is marginal in yielding tropospheric information. The scatter of points arises from computational inaccuracies, such as homing and possibly higher order effects. 


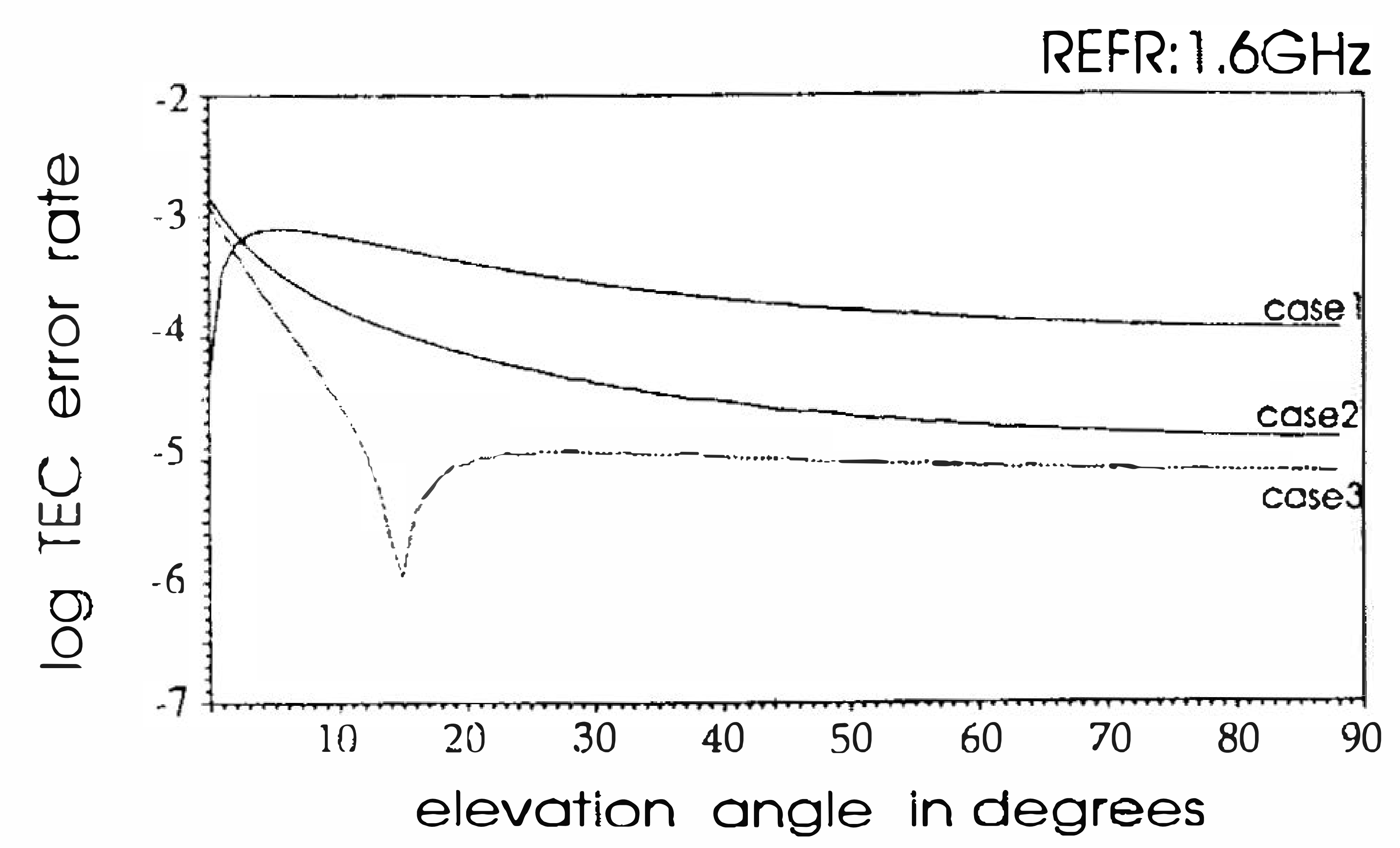

Fig. 3. The relative error in logarithmic scale of TEC computed by using formula (21) for the adopted model atmosphere as a function of the elevation angle. See text for choices of frequencies in the three cases. Note that for Case 1, there is at least three-digit accuracy for all elevation angles.

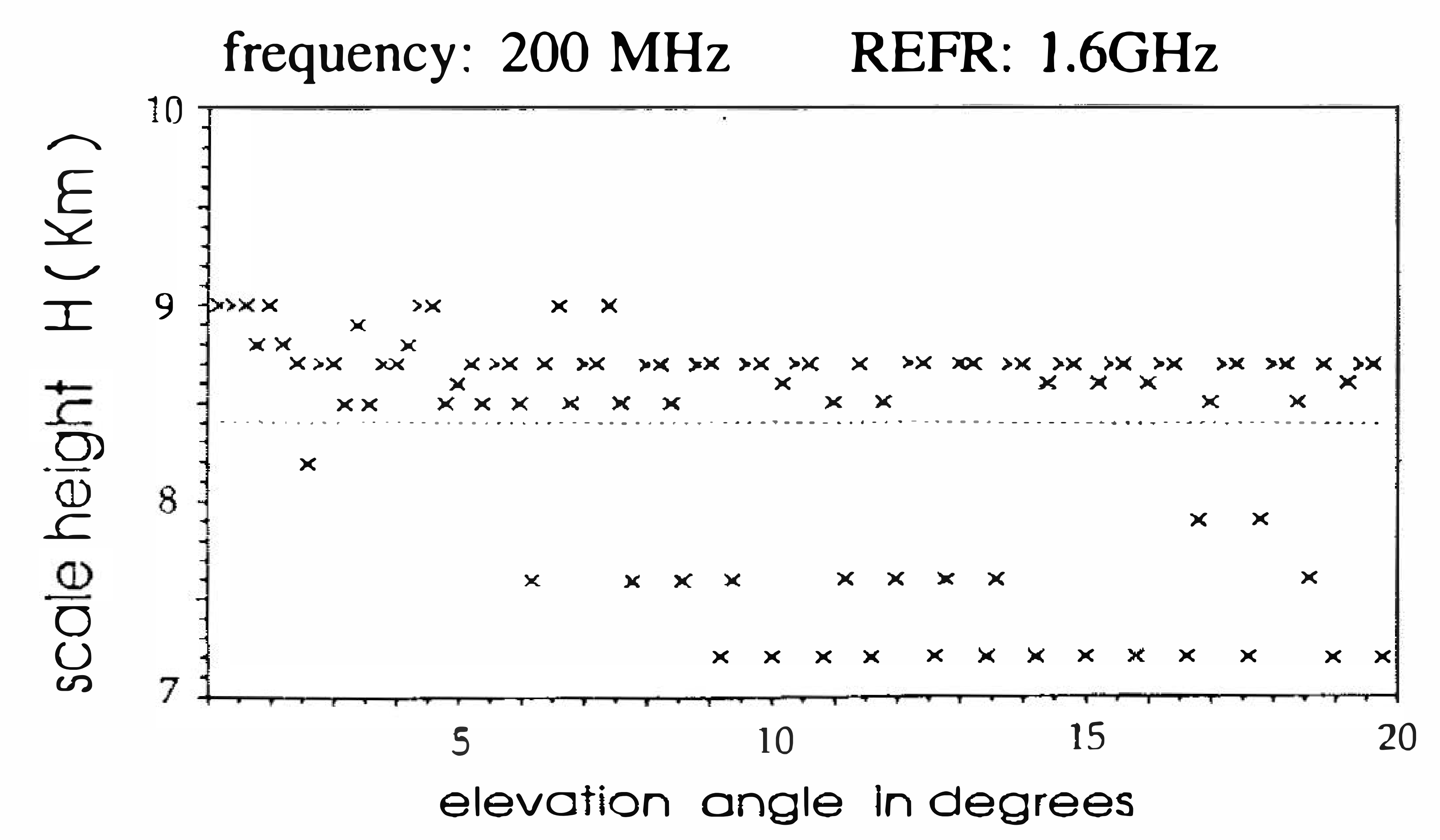

Fig. 4. The calculated scale height (indicated by crosses) by using the remaining dispersive phase difference between $200 \mathrm{MHz}$ and $1.6 \mathrm{GHz}$. The model adopts a scale height value of $8.4 \mathrm{~km}$ as indicated by the horizontal dashed line.

\section{DISCUSSION AND CONCLUSION}

The measurement technique investigated in this paper relies on phase measurements. Therefore, there are at least two problems that need to be addressed. The first has to do with the sensitivity of the measurement technique. For a number of reasons, the measured phase in the form of the output of a phase meter has jitters of a few degrees. What would a jittery phase mean to the accuracy of the TEC measurement? This is the sensitivity problem. The investigation by one of these authors (Yeh, 1994) shows that for a $5^{\circ}$ uncertainty in phase, the uncertainty in the TEC is less than one percent for all elevation angles for the frequency pair of $200 \mathrm{MHz}$ and $1.6 \mathrm{GHz}$. The uncertainty in the TEC increases as the lower frequency is increased from $200 \mathrm{MHz}$.

The second problem with the phase measurement has to do with the ambiguity problem ( Checcacci and de Giorgio, 1975). A phase is given by $\phi=2 k \pi+\phi^{\prime}, 0<\phi^{\prime}<2 \pi$. Here 
only $\phi^{\prime}$ is measured. The integer $k$ needs to be determined. Usually $k$ is large, making the determination of $k$ ambiguous. An auxiliary technique must be found that will determine $k$ . One such technique is the second phase difference technique (Burns and Fremouw,1970), by which the carrier frequency $f_{c}$ is modulated by a modulation frequency $f_{m}$. The phase difference between the upper side band and the carrier, and the phase difference between the carrier and the lower side band are first measured. The difference between these two differences is then calculated. By properly choosing $f_{m}$, the integer $k$ can be determined. In Taiwan, the TEC varies from $10^{16}$ electrons $/ m^{2}$ to $10^{18}$ electrons $/ m^{2}$ (Huang and Cheng, 1991; Huang, 1993). A suitable modulation frequency for a $200 \mathrm{MHz}$ carrier can be 5 to 10 MHz (Yeh, 1994).

In this investigation the adopted exponential tropospheric model and the Chapman ionospheric model are both oversimplified. The real troposphere is very complex, at times displaying temperature inversions. These inversions can trap the ray and modify the ray trajectory a great deal. Futhermore, the atmosphere may not be spherically stratified and may not be dry. The humidity in the earth's atmosphere is highly variable, and may introduce important effects. All these generalizations can make the computations very tedious but may also bring about some new phenomena.

With the use of simplified model atmospheres, it has been demonstrated how an accurate TEC can be measured by using the difference in the TEC at two lower frequencies to correct the TEC at a higher frequency, even at extremely low elevation angles. This high accuracy is paid for by requiring the satellite to emit at four frequencies. For an exponential troposphere, the tropospheric information contained in the phase data is difficult to extract with a high degree of accuracy.

Acknowledgements The authors wish to thank Professors Y. -H. Chu, Y. -W. Kiang and C. -W. Kuo for their comments on this work. This research is partially supported by a grant from the National Science Council under NSC83-0202-M-110-001.

\section{REFERENCES}

Aarons, J., 1982: Global morphology of ionospheric scintillation. Proc. IEEE, 70, 360-378.

Bhattacharyya, A., K. C. Yeh, and S. J. Franke, 1992: Deducing turbulence parameters from transionospheric scintillation measurements. Space Sci. Rev., 61, 335-386.

Burns, A. A., and E. J. Fremouw, 1970: A real-time correction technique for transionospheric ranging error. IEEE Trans. Ant. Propagat., AP-18, 785-790.

Checcacci, P. F., and M. T. de Giorgio, 1975: Note on the calibration of TEC curves from geostationary satellites. J. Atmos. Terr. Phys., 37, 219-221.

Davies, K., 1980: Recent progress in satellite radio beacon studies with particular emphasis on the ATS-6 radio beacon experiment. Space Sci. Rev., 25, 357-430.

Fan. D. J., and C. H. Liu, 1983: A morphological study of gigahertz equatorial scintillations in the Asia region. Radio Sci., 18, 241-252.

Garriott, O. K., A. V. da Rosa, and W. J. Ross, 1970: Electron content obtained from Faraday rotation and phase length variations. J. Atmos. Terr. Phys., 32, 705-727. 
Gorbunov, M. E., and A. S. Gurvich, 1993: Remote sensing of the atmosphere using a system of synchronously orbiting satellites. Radio Sci., 28, 595-602.

Hall, M., 1979: Effects of the troposphere on radio propagation. IEE Electromagnetic Waves Series 8, Peter Peregrinus Ltd., London, U.K.

Huang, Y. N., 1993: Ionospheric observations and research in Taiwan. In: F. -S. Kuo (Ed.), Low-Latitude Ionospheric Physics, COSPAR Colloquia Series Vol. 7, 27-39, Elsevier, Oxford, U.K.

Huang, Y. N., and K. Cheng, 1991: Ionospheric disturbances at equatorial anomaly crest region during the March 1989 magnetic storms. J. Geophys. Res., 96, 13953-13965.

Lawrence, R. S., C. G. Little, and H. J. A. Chivers, 1964: A survey of ionospheric effects upon earth-space radio propagation. Proc. IEEE, 52, 4-27.

Lee, M. -C (Ed.), 1992: Proc. Int'l Beacon Satellite Symposium, Cambridge, MA. 325pp.

Yeh, F. -R., 1994: A study of ionospheric and tropospheric effects for low elevation angle radio waves. M.S. thesis, Department of Electrical Engineering, National Sun Yat-sen University, Kaohsiung, Taiwan. 62pp. (in Chinese)

Yeh, K. C., and G. W. Swenson, Jr.,1961: Ionospheric electron content and its variations deduced from satellite observations. J. Geophys. Res., 66, 1061-1067.

Yeh, K. C., and C. H. Liu, 1982: Radio wave scintillations in the ionosphere. Proc. IEEE, 70, 324-360.

Yeh, K. C., and T. D. Raymund, 1991: Limitations of ionospheric imaging by tomography. Radio Sci., 26, 1361-1380. 